\title{
Ectopic Cushing's Syndrome secondary to Recurrent Thymic Neuroendocrine Carcinoma with Bilateral Ovarian Metastases: A Case Report
}

\author{
Eunice Yi Chwen Lau and Zanariah Hussein
}

Division of Endocrinology, Department of Medicine, Hospital Putrajaya, Federal Territory of Putrajaya, Malaysia

\begin{abstract}
Cushing's syndrome due to ectopic adrenocorticotrophic hormone (ACTH) secretion is uncommon, accounting for 9 to $18 \%$ of cases; approximately $10 \%$ of ACTH producing tumours are caused by thymic carcinomas. ${ }^{1}$ We describe a young lady who presented with Cushing's syndrome secondary to a primary neuroendocrine tumour (NET) arising from the thymus. She had surgical resection of her primary tumour with remission of her Cushing's syndrome however subsequently went on to have locoregional recurrence followed by distant metastases to her bilateral ovaries. She underwent 6 surgeries including bilateral adrenalectomy and had 3 cycles of chemotherapy over the course of the 8 years since her diagnosis. Due to the rarity and highly aggressive nature of this disease, we highlight the need for a multidisciplinary team approach and use of multiple modalities in the management of our patient. Timely use of bilateral adrenalectomy particularly in young patients is important to prevent further complications and facilitate other treatment modalities.
\end{abstract}

Key words: adrenalectomy, ectopic ACTH syndrome, Krukenberg tumour, neuroendocrine tumour, thymic tumour

\section{INTRODUCTION}

Thymic neuroendocrine tumours (TNETs) are rare tumours with an incidence of 0.02 per 100,000 population per year among Caucasians and 0.04 per 100,000 population per year among Asian or Pacific Islanders based on data from the SEER database. ${ }^{2}$ In the latest 2015 World Health Organisation classification, TNETs have been classified into 4 categories: Typical carcinoids (TC) (low grade or grade 1) and atypical carcinoids (AC) (intermediate grade or grade 2) which are well differentiated; large and small cell neuroendocrine carcinomas (high grade or grade 3 ) which are poorly differentiated. ${ }^{3}$ Data have suggested that Asian patients may have a predominance of AC with one study in Beijing showing AC in $60 \%$, TC in $10 \%$ and $27 \%$ were poorly differentiated. By comparison, the SEER database which comprised of $83 \%$ Caucasians, only $10 \%$ of patients were AC. ${ }^{4}$ Diagnosis may be challenging as onethird of patients are asymptomatic and are only identified on routine imaging. Others may present with mass effect due to compression of surrounding nerves or vessels. Carcinoid syndrome is rare, occurring in less than $1 \%$ of TNETS while approximately 40 to $50 \%$ can present with ectopic ACTH syndrome. ${ }^{4}$ Twenty five percent of patients are associated with multiple endocrine neoplasia 1 (MEN1) syndrome. ${ }^{4}$ As this is a rare condition, there is a lack of prospective trials or large series and cases are managed in a similar manner as broncho-pulmonary carcinoids. Ovarian metastases can occur in neuroendocrine tumours
(NETs) but they are uncommon and there is no current recommendation on their management in this situation. In this case report, we present a complex case of a young female with recurrent metastatic thymic neuroendocrine tumour with bilateral ovarian metastases and the multiple treatment modalities that were used over the years for her disease control.

\section{CASE}

A 23-year-old female of Indian ethnicity presented with hyperpigmentation, acne, progressive weight gain and irregular menses over 3 years. Clinically, she appeared cushingoid with truncal obesity and moon facies. She achieved menarche at age 13 and previous menses were regular. She had no history of past medical illness and no family history suggestive of MEN-1 syndrome. Her initial investigations performed at a private medical centre showed a markedly elevated adrenocorticotrophic hormone (ACTH) level of $221 \mathrm{mIU} / \mathrm{L}$ and positive high dose dexamethasone suppression test. Computed tomography of the thorax, abdomen and pelvis (CTTAP) showed an anterior mediastinal mass measuring $2.1 \times 2.8 \times 3.0 \mathrm{~cm}$, located behind the sternum and hyperplasia of the adrenal glands. She underwent surgical removal of her anterior mediastinal mass by a cardiothoracic surgeon at a private centre. Histopathology showed a moderately differentiated neuroendocrine tumour grade 2 with a mitotic index 5/10 hpf and Ki-67 
proliferative index of $10 \%$. The tumour stained positive for synaptophysin, chromogranin and ACTH and was negative for thyroid transcription factor-1 (TTF-1). Post-operatively, she displayed clinical resolution of her Cushing's syndrome with resumption of her regular menses and a Gallium-68 (Ga-68) DOTATOC positron emission tomography (PET)/CT scan 4 months post-operatively showed no evidence of somatostatin receptor (SSTR) avid disease.

A year post-surgery, she developed progressive weight gain and increasing ACTH levels. CTTAP showed recurrence with multiple solid nodules at the site of her previous surgery in the upper left mediastinum. Ga-68 DOTATOC PET/CT scan showed new focus of SSTR avid disease in the superior mediastinal node anterior to the innominate artery and multiple pre-vascular nodes near the previous surgical site. F18-Fluorodeoxyglucose (F18-FDG) PET/CT scan showed concordant uptake with the Ga-68 DOTATOC PET/CT scan. She had her second surgery approximately 3 years after her first surgery, with complete removal of her mediastinal tumour. Histopathology was consistent with an intermediate grade metastatic neuroendocrine tumour.

Her symptoms recurred a year after her second surgery. She developed diabetes and hypertension requiring medical therapy and was commenced on ketoconazole. CTTAP imaging showed an enlarged left supraclavicular node measuring $1.2 \mathrm{~cm}$ in the short axis diameter and reduction in size of her mediastinal lesion measuring $1.3 \times 2 \mathrm{~cm}$. Ga-68 DOTANOC PET/CT showed new focus of uptake at the left supraclavicular node with resolution of previous uptake at the superior mediastinum and pre-vascular nodes. Subsequently, $4 \frac{1}{2}$ years after her first surgery, an attempt was made to remove the left supraclavicular lymph node. However, adhesions to the surrounding structures resulted in inability to completely remove the node.

Post-operatively, she had persistent Cushing's syndrome. Metyrapone was added to her therapy, nevertheless she continued to have difficult to control diabetes, hypokalaemia and progressive cushingoid features. CTTAP imaging approximately $1 \frac{1}{2}$ years after her $3^{\text {rd }}$ surgery showed multiple rounded lobulated lesions along the right postero-lateral uterine body largest measuring $4.6 \times 4.4 \times 5.2 \mathrm{~cm}$. There was no significant change in the left supraclavicular lesion. F18-FDG PET/CT scan showed uptake in both the supraclavicular and pelvic lesion, while Ga-68 DOTANOC PET/CT scan showed uptake in the supraclavicular lesion and right level II cervical nodes and anterior mediastinal node.

At this point decision was made for bilateral adrenalectomy (5 $1 \frac{1}{2}$ years after first surgery) for management of her hypercortisolism. Prior to adrenalectomy, she required $3000 \mathrm{mg}$ of metyrapone, $400 \mathrm{mg}$ ketoconazole, $200 \mathrm{mg}$ spironolactone, $160 \mathrm{mg}$ valsartan, $10 \mathrm{mg}$ amlodipine, 2000 $\mathrm{mg}$ metformin, $120 \mathrm{mg}$ of modified release gliclazide, $10 \mathrm{U}$ of intermediate acting insulin, $20 \mathrm{mg}$ atorvastatin, $1200 \mathrm{mg}$ potassium chloride and 1000 IU cholecalciferol per day.

Post-operatively, she had resolution of her Cushing's features, was able to stop all her potassium supplements, antihypertensive and antidiabetic medications and was put on glucocorticoid and mineralocorticoid replacement.
However, she continued to have increasing hyperpigmentation (Figure 1) and CTTAP imaging surveillance 6 months after her surgery showed increase in size of her uterine lesion likely right adnexal in origin measuring $5.8 \times 5.2 \times 5.2 \mathrm{~cm}$ in size (Figure 2A). Ga-68 DOTANOC PET/CT and F18-FDG PET/CT imaging were performed showing predominantly FDG avid disease (Figure 2B and $2 \mathrm{C}$ ), hence she was deemed unsuitable for peptide receptor radionuclide therapy (PRRT). Following multidisciplinary team discussion, she was recommended for surgery to remove the adnexal lesion. She was offered bilateral salphingoopherectomy and hysterectomy and the implications were discussed in terms of long-term gonadal function and bone health. She opted for a more conservative approach and underwent laparotomy, right salphingoopherectomy, left cystectomy, omentectomy and appendectomy.

Intra-operative findings showed a right large ovarian tumour measuring $10 \times 10 \mathrm{~cm}$ adherent to the right pelvic wall and 2 cysts in the left ovary measuring $1 \times 1 \mathrm{~cm}$ and $2 \times 2 \mathrm{~cm}$ (Figure 3A). Histopathology showed high grade metastatic neuroendocrine carcinoma with a Ki-67 proliferative index of $10 \%$, mitotic figures of $>10 / 2 \mathrm{~mm}^{2}$ and presence of capsular breach, lymphovascular invasion in both right and left ovaries. Marked improvement in ACTH levels were noted after surgery (Figure 4). Prior to surgery she continued to have her normal periods but was amenorrhoeic after. She was also given 3 cycles of chemotherapy with etopside $\left(100 \mathrm{mg} / \mathrm{m}^{2}\right.$ Day 1 to 3 ) and cisplatin $\left(25 \mathrm{mg} / \mathrm{m}^{2}\right.$ Day 1 to 3 ). However, CTTAP at 6 months showed progression of her left adnexal mass and ACTH levels continued to increase (Figure 4). She went on to have extrafascial hysterectomy and left salphingoopherectomy 8 months after the $5^{\text {th }}$ surgery. Intra-operative findings showed a 6-week size uterus, a 3x3 $\mathrm{cm}$ left ovarian tumour with intact capsule and a ruptured right sided pelvic wall tumour measuring $5 \times 4 \mathrm{~cm}$ (Figure 3B). Left ovarian histopathology was consistent with a high-grade neuroendocrine tumour (mitosis $>10 / 2 \mathrm{~mm}^{2}$ ).

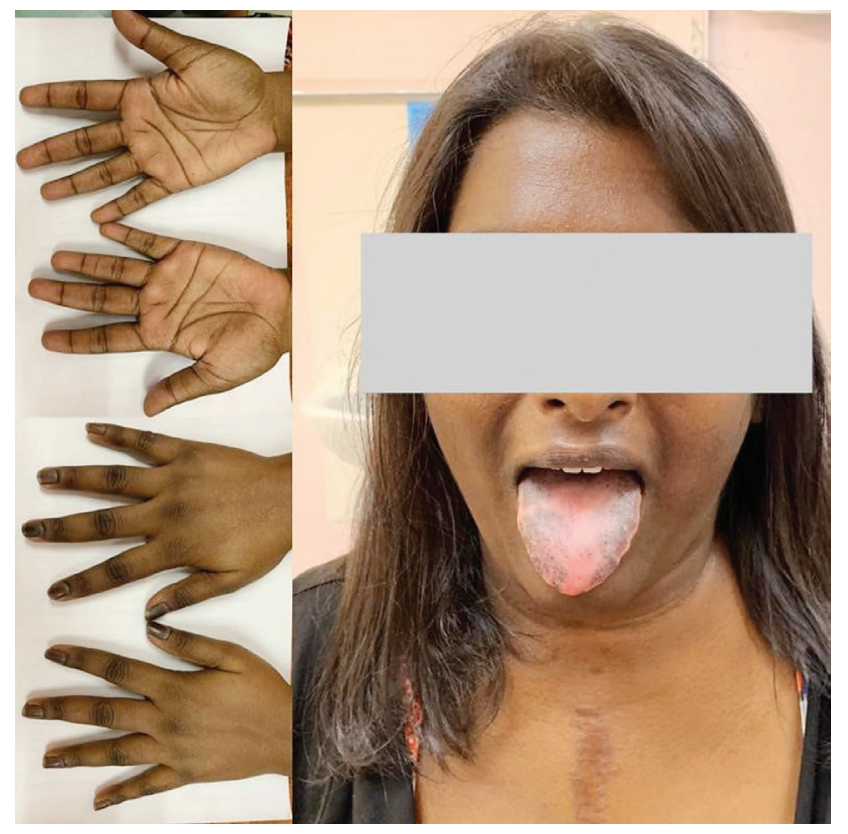

Figure 1. ACTH induced hyperpigmentation. 

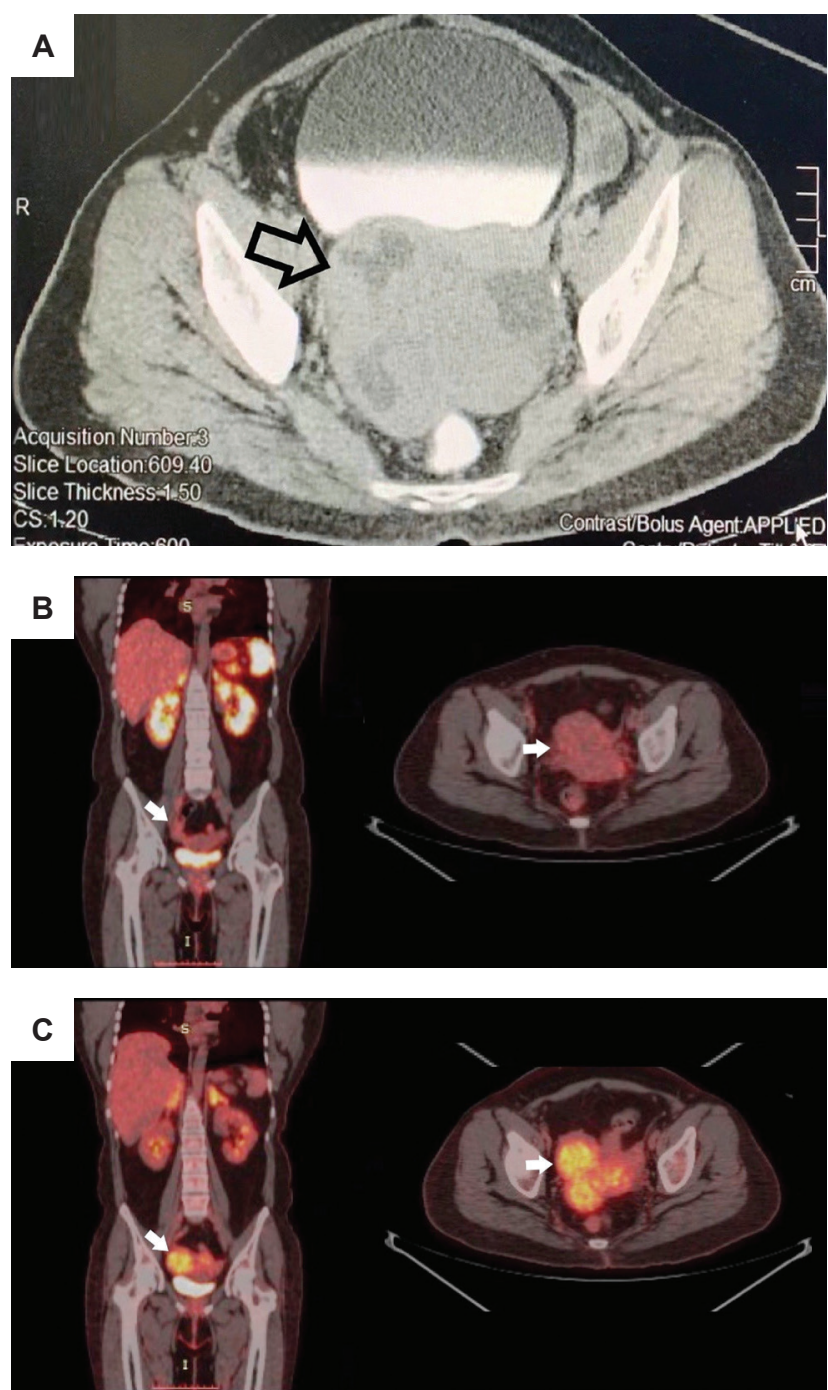

Figure 2. (A) CT image showing heterogenous multilobulated pelvic lesion; (B) Ga-68 DOTANOC PET-CT showing pelvic lesion with no SSTR avid disease. (C) FDG PETCT showing FDG hypermetabolism of the pelvic lesion.

After surgery, her ACTH levels reduced to $49.5 \mathrm{pmol} / \mathrm{L}$ and the trend is demonstrated in Figure 4. She developed symptoms of flushing and fatigue despite adequate glucocorticoid and mineralocorticoid replacement and was started on conjugated oestrogen $0.625 \mathrm{mg}$ and medroxyprogesterone $5 \mathrm{mg}$ daily. She is currently still followed-up at our centre and remains positive with good family support.

\section{DISCUSSION}

TNETs consist of $2 \%$ of all mediastinal tumours and $5 \%$ of all thymic tumours, making ectopic ACTH secreting TNETs exceedingly rare. TNETs usually present around the $5^{\text {th }}$ decade and have a male predominance. ${ }^{2,5}$ Comparatively, TNETs associated with ectopic ACTH syndrome appear to present younger, around 21 to 35 years of age with hyperpigmentation being a prominent feature. ${ }^{6,7}$ They also appear to have poorer outcomes, although it is uncertain whether this is due primarily to the metabolic complications associated with Cushing's syndrome or more aggressive tumour behaviour. ${ }^{6}$
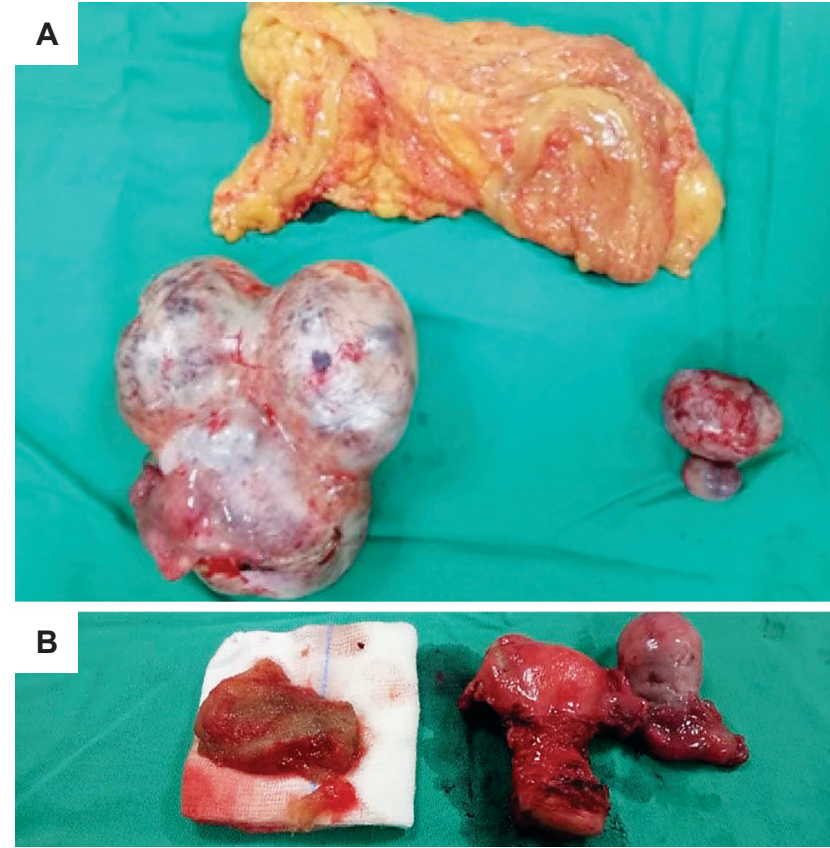

Figure 3. (A) Large right ovarian tumour, 2 left ovarian cysts and omentum; (B) Ruptured right pelvic tumour, uterus with left ovarian tumour.

A recent study of ectopic ACTH secreting TNETs in China showed median overall survival of 41 months and progression free survival of 28 months with significant improvement after complete resection. ${ }^{7}$ There appears to be wide heterogeneity in the tumours, while some may be rapidly progressive, others are more indolent and may recur up to 8 years after surgery..$^{5,6}$

Our patient achieved clinical and biochemical remission after initial tumour resection. However, her disease subsequently progressed over the period of several years requiring repeat surgery followed by adrenalectomy when the tumour became unresectable. Ovarian metastases while uncommon, can occur in TNETs as shown in this case. Most of the treatment decisions for this patient were made after multidisciplinary discussion with oncologists, surgeons, endocrinologists, radiologists, and gynaecologists. As there are no clear guidelines on the management of this disease, a multidisciplinary approach is important, and we highlight the multiple treatment modalities and review the evidence for their use.

In the past TNETS have been managed in a similar manner to bronchopulmonary carcinoids and were classified and graded as a single class of tumours. However, in recent years they have been acknowledged as having their own distinct behaviour and genetic background. Unlike their lung counterparts, TNETs have a more aggressive clinical course and frequently metastasize to lymph nodes, bone and lung. ${ }^{8,9}$ Less commonly, metastases have been reported in widespread locations including the oesophagus, chest wall, liver, brain, pancreas, kidney and adrenal gland..$^{8,9}$

In our patient, the tumour metastasized to both ovaries. This case is unique as our patient had bilateral ovarian metastases from a TNET which showed evidence of ACTH production. There has been report of ACTH secreting 


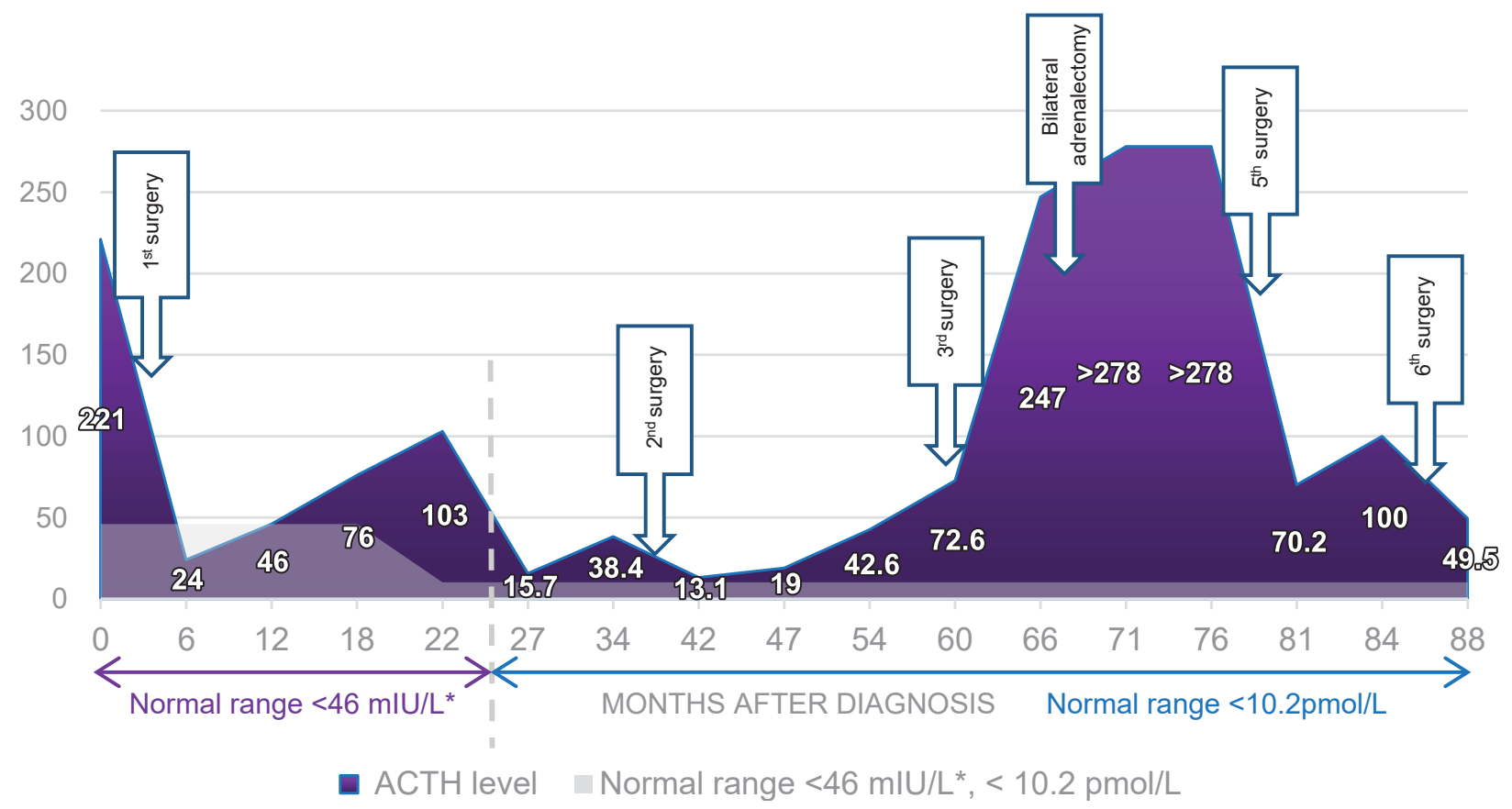

Figure 4. ACTH trend since diagnosis and corresponding normal range. Difference in ACTH cut-offs before and after 22 months was due to a change in assay used.

pancreatic NET presenting with bilateral ovarian metastasis but to our knowledge there is no published report of a functioning thymic NET with ovarian metastases. ${ }^{10}$ Generally thymic malignancies metastasize to intrathoracic sites first, with abdominal sites being involved later during the disease. ${ }^{11}$ Liver metastasis were the most common site of extra-thoracic metastasis with ovarian metastasis being rare. ${ }^{11}$ In neuroendocrine tumours, ovarian metastases were estimated to occur in approximately $2 \%$ patients and usually originated from midgut tumours predominantly the small bowel. ${ }^{12,13}$ They were usually bilateral, unlike primary ovarian lesions which were unilateral and could range from microscopic foci up to large tumours of 9.5 $\mathrm{cm}$ like our case. ${ }^{13}$ Previous reports have suggested that patients usually had well-differentiated NETs and $76 \%$ had synchronous liver and peritoneal metastases (including mesentery and omentum). ${ }^{13}$

Radical surgery has been widely agreed as the only option for cure. However, this may not be feasible in all patients as more than $50 \%$ have locally advanced disease or metastases at presentation. Even after radical surgery, recurrence is common as demonstrated by our case. Studies have shown that the most important independent prognostic factor is the completeness of resection whereby those who achieved R0 resection had improved overall survival compared to those who did not. ${ }^{5,7}$ Early stage tumours have also been reported to survive longer and have less recurrence. ${ }^{5}$ On the other hand, tumour histology did not appear to have significant prognostic implication. For recurrent or metastatic disease surgery may be considered if feasible. For primary ovarian NETs prophylactic omentectomy and hysterectomy have been performed as adapted from the management of ovarian cancer although it is uncertain whether this provides any additional benefit. ${ }^{14}$ Our patient was initially offered bilateral salphingoopherectomy and hysterectomy however she opted for a more conservative approach. Despite adjuvant chemotherapy, her disease continued to progress, and she subsequently required repeat surgery. This led to improvement in her ACTH levels. During this surgery, it was noted that the pelvic tumour had ruptured, likely leading to intraperitoneal seeding. This has been shown to greatly increase the risk of relapse in epithelial ovarian cancers. ${ }^{15}$ Hence, she will require careful monitoring for relapse with $\mathrm{ACTH}$ levels and imaging as well as long-term female gonadal hormone replacement on top of her adrenal hormone replacement.

For tumours that are metastatic or unresectable chemotherapy and radiotherapy may play a role although clear evidence is lacking. One study showed possible benefit of adjuvant therapy on progression free survival but not for overall survival ${ }^{7}$ while another study failed to show any benefit at all $^{5}$ as was the case in our patient. Patients that require adjuvant therapy probably had poorer prognosis and more extensive disease hence more research is needed to clearly evaluate this area.

In our patient, there was also difficulty in managing the metabolic complications of her Cushing's syndrome. Similar to previous reports, tumour recurrence was associated with re-development of Cushing's syndrome. ${ }^{6}$ Management of hypercortisolism is important and can be managed medically with drugs such as ketoconazole, metyrapone and less commonly mitotane in view of its serious side effects. In ectopic ACTH secreting neuroendocrine tumours, bilateral adrenalectomy is usually reserved for situations as in our patient whereby the primary tumour cannot be completely removed, have local recurrence or metastatic disease. The other instance would be when the primary tumour is occult and remains unidentified during follow-up. One study showed improvement in metabolic and adverse events (infection, fracture, thrombosis) scores in patients with refractory ACTH dependant Cushing's 
who underwent bilateral adrenalectomy compared to those who used steroidogenesis inhibitors alone. ${ }^{16}$ Hence, early bilateral adrenalectomy should be considered in those with reasonable life expectancy from their primary tumour as this may facilitate surgical resection or other treatment modalities in initially unresectable tumours and prevent further adverse complications.

\section{CONCLUSION}

ACTH producing TNET is an aggressive disease, and a multidisciplinary team approach is needed. Firstline treatment is surgery, especially in young patients. There is also the challenge of uncontrolled Cushing's and early adrenalectomy may be considered to optimize management. Young patients may have a prolonged disease course like our patient, requiring multiple treatment modalities as the disease progresses.

\section{Ethical Consideration}

Patient consent was obtained before submission of the manuscript.

\section{Statement of Authorship}

Both authors certified fulfilment of ICMJE authorship criteria.

\section{Author Disclosure}

Both authors declared no conflict of interest.

\section{Funding Source}

None.

\section{References}

1. Wajchenberg BL, Mendonca BB, Liberman B, et al. Ectopic adrenocorticotropic hormone syndrome. Endo Rev. 1994;15(6):752-87. PMID: 7705280. https://doi.org/10.1210/edrv-15-6-752.

2. Gaur P, Leary C, Yao JC. Thymic neuroendocrine tumors: A SEER database analysis of 160 patients. Ann Surg. 2010;251(6):1117-21. PMID: 20485130. https://doi.org/10.1097/SLA.0b013e3181dd4ec4.

3. Travis WD, Brambilla E, Burke AP, Marx A, Nicholson AG, eds. WHO Classification of Tumours of the Lung, Pleura, Thymus and Heart, 4th ed. Lyon, IARC Press; 2015. https://publications.iarc.fr/Book-AndReport-Series/Who-Classification-Of-Tumours/WHO-ClassificationOf-Tumours-Of-The-Lung-Pleura-Thymus-And-Heart-2015.

4. Jia R, Sulentic P, Xu JM, Grossman AB. Thymic neuroendocrine neoplasms: Biological behaviour and therapy. Neuroendocrinol. 2017;105(2):105-14. PMID: 28355610. https://doi.org/10.1159/000472255.
5. Filosso PL, Yao X, Ahmad U, et al. Outcome of primary neuroendocrine tumors of the thymus: A joint analysis of the International Thymic Malignancy Interest Group and the European Society of Thoracic Surgeons databases. J Thorac Cardiovasc Surg. 2015;149(1):103-9.e2. PMID: 25308116. https://doi.org/10.1016/j.jtcvs.2014.08.061.

6. Neary NM, Lopez-Chavez A, Abel BS, et al. Neuroendocrine ACTHProducing tumor of the thymus-Experience with 12 patients over 25 years. J Clin Endocrinol Metab. 2012;97(7): 2223-30. PMID: 22508705. PMCID: PMC3387392. https://doi.org/10.1210/jc.2011-3355.

7. Chen YY, Li SQ, Liu HS, et al. Ectopic adrenocorticotropic hormone syndrome caused by neuroendocrine tumors of the thymus: 30 year experience with 16 patients at a single institute in the People's Republic of China. Onco Targets Ther. 2016;9:2193-201. PMID 27217765. PMCID: PMC4853161. https://doi.org/10.2147/OTT.S100585.

8. Moran CA, Suster S. Neuroendocrine carcinomas (carcinoid tumor) of the thymus. A clinicopathologic analysis of 80 cases. Am J Clin Pathol. 2000; 114(1):100-10. PMID: 10884805. https://doi.org/10.1309/ 3PDN-PMT5-EQTM-H0CD.

9. Araki T, Sholl LM, Hatabu H, Nishino M. Radiological features and metastatic patterns of thymic neuroendocrine tumours. Clin Radiol. 2018;73(5):479-44. PMID: 29310810. https://doi.org/10.1016/ j.crad.2017.11.025.

10. Oberg KC, Wells K, Seraj IM, et al. ACTH-secreting islet cell tumor of the pancreas presenting as bilateral ovarian tumors and Cushing's Syndrome. Int J Gynecol Pathol. 2002;21(3):276-80. PMID: 12068175. https://doi.org/10.1097/00004347-200207000-00012.

11. Khandelwal A, Sholl LM, Araki T, et al. Patterns of metastasis and recurrence in thymic epithelial tumours: Longitudinal imaging review in correlation with histological subtypes. Clin Radiol. 2016;71(10): 1010-7. PMID: 27267746. PMCID: PMC5010483. https:// doi.org/10.1016/j.crad.2016.05.007.

12. Pavel M, Grossman A, Arnold R, et al. ENETS consensus guidelines for the management of brain, cardiac and ovarian metastases from neuroendocrine tumors. Neuroendocrinol. 2010;91:326-32. PMID: 20453466. https://doi.org/10.1159/000287277.

13. Strosberg J, Nasir A, Cragun J et al. Metastatic carcinoid tumor to the ovary: A clinicopathologic analysis of seventeen cases. Gynecol Oncol. 2007;106(1):65-8. PMID: 17475313. https://doi.org/10.1016/ j.ygyno.2007.02.034.

14. Sehouli J, Woopen $\mathrm{H}$, Pavel $\mathrm{M}$ et al. Neuroendocrine neoplasm of the ovary: A retrospective study of the North Eastern German Society of Gynecologic Oncology (NOGGO). Anticancer Res. 2016;36(3):1003-9. PMID: 26976990.

15. Tan DSP, Agarwal R, Kaye SB. Mechanisms of transcoelomic metastasi in ovarian cancer. Lancet Oncol. 2006;7(11):925-34. PMID: 17081918 https://doi.org/10.1016/S1470-2045(06)70939-1.

16. Morris LF, Harris RS, Milton DR, et al. Impact and timing of bilateral adrenalectomy for refractory ACTH-dependent Cushing's syndrome. Surgery. 2013;154(6):1174-83. PMID: 24383115. PMCID: PMC4271839. https://doi.org/10.1016/j.surg.2013.06.017.

Authors are required to accomplish, sign and submit scanned copies of the JAFES Author Form consisting of: (1) Authorship Certification, that authors contributed substantially to the work, that the manuscript has been read and approved by all authors, and that the requirements for authorship have been met by each author; (2) the Author Declaration, that the article represents original material that is not being considered for publication or has not been published or accepted for publication elsewhere, that the article does not infringe or violate any copyrights or intellectual property rights, and that no references have been made to predatory/suspected predatory journals; (3) the Author Contribution Disclosure, which lists the specific contributions of authors; (4) the Author Publishing Agreement which retains author copyright, grants publishing and distribution rights to JAFES, and allows JAFES to apply and enforce an Attribution-Non-Commercial Creative Commons user license; and (5) the Conversion to Visual Abstracts (*optional for original articles only) to improve dissemination to practitioners and lay readers Authors are also required to accomplish, sign, and submit the sioned ICMJE form for Disclosure of Potential Conflicts of Interest. For original articles, authors are required to submit a scanned copy of the Ethics Review Approval of their research as well as registration in trial registries as appropriate. For manuscripts reporting data from studies involving animals, authors are required to submit a scanned copy of the Institutional Animal Care and Use Committee approval. For Case Reports or Series, and Images in Endocrinology, consent forms, are required for the publication of information about patients; otherwise, appropriate ethical clearance has been obtained from the institutional review board. Articles and any other material published in the JAFES represent the work of the author(s) and should not be construed to reflect the opinions of the Editors or the Publisher. 